\title{
Responsabilidad de la industria farmacéutica sobre la inversión en investigación de las enfermedades olvidadas. Propuesta de mejora
}

\author{
Responsibility of the pharmaceutical industry on research \\ investment in neglected diseases. Proposal for improvement
}

XAVIER CASAS ROMA*

\begin{abstract}
Resumen: Millones de personas sufren o mueren debido a las enfermedades olvidadas. Sostengo que la industria farmacéutica debería invertir más de lo que actualmente hace para combatirlas. En este artículo se discute la mayor implicación que la industria farmacéutica debe tener con la investigación frente a las enfermedades olvidadas, comparando mi propuesta a otros esquemas alternativos como los de Pogge, Reiss o de Winter.

Palabras clave: Enfermedades olvidadas, investigación biomédica, industria farmacéutica, ética investigación, responsabilidad social corporativa.
\end{abstract}

\begin{abstract}
Millions of people suffer or die from neglected diseases. I contend that the pharmaceutical industry should invest more than it actually does in fighting them. In this paper I argue for a more significant involvement of the industry in the research against neglected diseases, comparing my proposal to alternative schemes such as those of Pogge, Reiss or de Winter.

Keywords: Neglected diseases, biomedical research, pharmaceutical industry, research ethics, and corporate social responsibility.
\end{abstract}

La inversión en investigación biomédica (BMR, del inglés BioMedical Research) es motivo de un debate ético que afecta al ámbito académico, la industria privada, los gobiernos y, por extensión, a los ciudadanos. Campos como la clonación, la investigación con células madre, los organismos modificados genéticamente, el desarrollo de nuevos fármacos y vacunas, la experimentación animal y humana son sólo algunos de los múltiples ejemplos de los distintos ámbitos de este debate. En la mayoría de estos campos el debate ético existente pretende moderar y orientar los avances y los límites, si los hubiera, de la investigación. La industria farmacéutica es el centro del debate sobre el que gira la cantidad y destino de los recursos que se deben invertir en el desarrollo de nuevos fármacos para las enfermedades

Fecha de recepción: 25/04/2013. Fecha de aceptación definitiva: 10/12/2014.

* Universidad Nacional de Educación a Distancia, adscrito al Departamento de Lógica, Historia y Filosofía de la Ciencia. Líneas de investigación: el papel de la industria farmacéutica en la sociedad actual, especialmente en los países en vías de desarrollo y la responsabilidad de los distintos agentes implicados en la alimentación (proteina animal), también en los países en vías de desarrollo. Correo electrónico: xcasasroma@gmail.com 
que afectan a los más desfavorecidos. Los detractores de la política actual planteada por la industria farmacéutica argumentan que la investigación se está dejando llevar por los beneficios económicos en lugar de por las mejoras que los beneficios médicos y sanitarios puedan aportar a la humanidad (Reiss, 2009).

El presente trabajo aborda uno de los campos del debate que, probablemente, menor repercusión tiene, tanto a nivel científico como a nivel mediático: la inversión en la investigación de las enfermedades olvidadas (EO) y la responsabilidad ética que la industria farmacéutica tiene en este campo.

\section{Definición del concepto «Enfermedad Olvidada»: ¿De qué estamos hablando?}

Si bien existen numerosas publicaciones que tratan temas directamente relacionados con las «enfermedades olvidadas» (EO), la definición de las mismas difiere ligeramente según los autores y/o las instituciones que publican sobre ellas. Así por ejemplo la Organización Mundial de la Salud (OMS) a través de su Directora General Dra. Margaret Chan, define las Enfermedades Tropicales Olvidadas (ETO) ${ }^{1}$ del modo siguiente: «Un grupo de enfermedades que están fuertemente ligadas a la pobreza, ambientes pobres y que se desarrollan con gran facilidad en áreas tropicales, donde suelen co-existir. Muchas de ellas son enfermedades antiguas que han afectado a la humanidad durante siglos. Si bien han desaparecido de las partes del mundo más desarrolladas gracias a la mejora de las condiciones de higiene, todavía afectan la vida de más de 1.000.000.000 de personas en áreas remotas rurales o en los suburbios de las ciudades» (WHO report 2010).

Se puede considerar que las EO comparten una serie de características comunes que las definen. La OMS ha detectado siete (WHO report 2010):

- Son indicadores de pobreza y recursos limitados.

- Afectan a poblaciones con poca relevancia política.

- No se transmiten de forma masiva.

- Son causa de estigmas y discriminación, especialmente en niñas y mujeres.

- Tienen un impacto importante en la morbilidad y la mortalidad.

- Son relativamente poco consideradas a nivel de investigación.

- Pueden ser controladas, prevenidas y posiblemente eliminadas utilizando soluciones ya existentes y efectivas (por ejemplo, con medidas de vigilancia y control para erradicar el vector -mosquito- que transmite el dengue).

Diversos autores, por ejemplo Reiss (2009), establecen una relación directa entre el término «olvidada» con la falta de inversión en Investigación y Desarrollo (I+D). Es decir, para este grupo de autores, una enfermedad se puede calificar de «olvidada» cuando los recursos invertidos en $\mathrm{I}+\mathrm{D}$ es muy inferior a la cantidad que debería invertirse teniendo en cuenta las consecuencias (mortalidad, pero también morbilidad) que la enfermedad genera, especialmente en los países en vías de desarrollo.

1 Como demuestra el ejemplo de la OMS, frecuentemente se usan como sinónimos los conceptos de «enfermedad olvidada» y «enfermedad tropical olvidada», puesto que en la práctica totalidad de los casos, las EO se centran en zonas tropicales. 
Se obvian en esta definición y en este artículo otro tipo de enfermedades que, si bien son también enfermedades «olvidadas» en términos de inversión en investigación, no cumplen con los requisitos y características señalados en este párrafo; por ejemplo, las enfermedades minoritarias que pueden afectar también algunos (pocos) individuos de los países desarrollados.

\section{Responsabilidad ética de la industria farmacéutica en la investigación biomédica de las enfermedades emergentes}

La relación de la industria farmacéutica con los países en vías de desarrollo y, especialmente, con la investigación biomédica (BMR), es motivo de múltiples publicaciones que la califican como deficiente a nivel epistemológico, moral, y socio-económico (Reiss, 2010). Es evidente que el gran negocio de la industria farmacéutica se localiza en los países occidentales y se orienta a «enfermedades occidentales», por ejemplo el cáncer, enfermedades cardiovasculares, degenerativas etc. (Reiss, 2009). De hecho, la mayoría de productos comercialmente disponibles en los países en vías de desarrollo son productos antiguos desarrollados por la industria de países occidentales (o bien por la propia industria local en colaboración con la industria occidental) y que data de la época de las colonias, a principios del siglo XX (Trouiller, 2002). En la actualidad y, probablemente debido a razones de moralidad pero también debido a la crítica de la opinión pública, el esfuerzo que la industria está realizando en los países en vías de desarrollo parece que se está incrementando. Así por ejemplo, entre el año 2000 y 2006, la industria mundial invirtió un total de 6,7 billones de dólares en estos países, incluyendo donaciones de medicinas, vacunas, instrumental, equipos de diagnóstico y labores de formación y educación (IFPMA, 2007). Éstos datos son armas de doble filo, por ejemplo: el producto gratis que se envía a estos países frecuentemente responde a intereses de la propia industria para mantener un precio del medicamento «global», sin grandes diferencias entre zonas (Pollock, 2011); de ésta forma, las estadísticas de ventas aparecen maquilladas y distribuidas por todo el mundo, pero realmente sólo los países con mayor poder adquisitivo pueden sostener unas ventas reales con un precio inflado del medicamento. Ofrecer un producto sensiblemente más económico en un territorio determinado, facilitaría el comercio ilegal entre países, además de desestabilizar el precio «adecuado» para aquellos mercados que pueden permitírselo (Oxfam, Save the Children, VSO, 2002). Además, muchos de estos países son elegidos para realizar ensayos con fármacos o vacunas de forma más rápida, con menor coste y sin la presión mediática y legal existente en los países occidentales (Global Issues, 2011).

Si bien la tendencia actual por parte de la industria farmacéutica, al menos a nivel de comunicación, se presume que es acercarse más al problema de la inversión en investigación de las EO, las ideas y buenas intenciones deben plasmarse en proyectos reales y cuantificables, no sólo en el sentido de inversión monetaria de «X» euros en un proyecto determinado, sino también cuantificables a nivel de resultados obtenidos en el país o países destino. Se trata, simplemente, de dejar atrás el modelo de «filantropía», entendido como donaciones y trabajos de tipo voluntario, para establecer un nuevo modelo en el que la búsqueda activa de la mejora real de la investigación de las EO sea la idea central sobre la que se articule la nueva relación de la industria farmacéutica y resto de sectores implicados con las EO. 
La responsabilidad social corporativa (RSC), es un concepto que ha suscitado intensos debates, especialmente cuando se ha aplicado a las grandes empresas o corporaciones transnacionales (CTN). En un excelente trabajo, Leisinger (Leisinger, 2005) define la responsabilidad social como «la responsabilidad frente a la sociedad en general» y distingue esta responsabilidad, especialmente para la industria farmacéutica, en 3 partes diferenciadas según lo que la sociedad espera de la propia industria:

- Lo «requerido» por la sociedad, o el «debe hacer» de la industria: aquí caerían acciones socialmente responsables que la sociedad exige a la industria, por ejemplo, condiciones mínimas y decentes de trabajo, asegurar la puesta en el mercado de productos con una calidad mínima etc.

- Lo «esperado» por la sociedad, o el «debería hacer» de la industria: aquí entrarían acciones que la sociedad espera de las compañías, como por ejemplo una política medioambiental adecuada.

- Lo «deseado» por la sociedad, o el «puede hacer» de la industria: aquí caen las acciones en relación con la filantropía corporativa, por ejemplo las donaciones de productos etc.

La contribución de la industria farmacéutica en la investigación de las EO queda englobada en el tercero de los grupos; es decir, la sociedad desea que la industria pueda implicarse en este sentido.

Algunos organismos que trabajan con los más desfavorecidos, como son Oxfam, Save the Children y Voluntary Service Overseas -VSO (Oxfam, 2002), retan a la industria farmacéutica a adoptar un nuevo concepto de RSC que se enfoque en las crisis sanitarias que afectan a los países en vías de desarrollo. En el informe que presentan, se reconocen los considerables avances conseguidos en este ámbito en los últimos años, aún insuficientes, y se pretende estimular a la industria a ampliar el interés y los recursos destinados a las enfermedades infecciosas que afectan a los países en vías de desarrollo. Para conseguirlo, proponen a la industria farmacéutica una serie de compromisos en cada uno de los 5 puntos estratégicos siguientes: precio; patentes; conjunto de iniciativas públicas-privadas; Investigación y Desarrollo y uso apropiado de los medicamentos. En cada uno de los 5 puntos, el informe recomienda una serie de compromisos demostrables que cumplen con lo que se espera de la RSC de la industria farmacéutica con los países en vías de desarrollo.

Una vez revisada la compleja relación de intereses y fuentes de financiación que tejen la red sobre la que las empresas farmacéuticas desarrollan su negocio, la pregunta que surge es la siguiente: ¿tiene la industria farmacéutica una responsabilidad social frente a las enfermedades que afectan a los más pobres y, en caso de ser así, es esta responsabilidad mayor que la de otros sectores?

Acción-Omisión: dos formas distintas de enfocar el problema de la RSC. La responsabilidad social de las grandes compañías ha sido ligada históricamente con las «acciones» que la compañía toma y las consecuencias que tiene como resultado de la aplicación de sus políticas, sobre todo a nivel mundial. Así pues, los principales debates éticos sobre el comportamiento de las grandes corporaciones, según Naciones Unidas (Leisinger, 2005), se han centrado básicamente en las acciones que las empresas han tomado en relación a 4 ámbitos diferenciados: 
Derechos humanos; normas laborales; medioambiente y anti-corrupción. Por supuesto, existen otros muchos ámbitos en los que se puede debatir la ética de las empresas, pero estos últimos han suscitado menos interés que los anteriores, especialmente aquellos ámbitos de debate que afectan a los países en vías de desarrollo debido a la poca importancia relativa y peso político que los países pobres tienen en el mercado global (United Nations, 1999). Resulta razonable, por ejemplo, exigir a una empresa (farmacéutica, química, automovilística, petrolera etc.) que reinvierta una parte de sus beneficios en asegurar una política medioambiental adecuada, que no dañe el ecosistema del lugar de producción y, que en caso de hacerlo, se encargue de reducir al máximo los efectos no deseados que la propia actividad productiva genera. Es evidente que las acciones que una empresa lleva a cabo, especialmente las CTN, resultan éticamente más fáciles de valorar. Pero, ¿qué ocurre con las acciones que las empresas no han llevado a cabo y podrían (o deberían) haber llevado a cabo? ¿Qué ocurre con la «omisión» de ciertas decisiones o políticas que pueden tener un efecto, por ejemplo, a nivel de la salud de la población mundial? En un excelente trabajo, Holly Lawford-Smith (Lawford-Smith, 2012) alerta de la distinción que la sociedad hace entre acciones y omisiones. Si bien ambos casos (acción - omisión) pueden ser moralmente reprobables, lo cierto es que en el segundo de los casos resulta menos grave o punible a ojos de la población en general y, por tanto, menos susceptible de ser condenado y sometido a mejora gracias al empuje que parte de la sociedad.

Cuando la omisión de la industria farmacéutica (no prestar la atención necesaria a la investigación y desarrollo de nuevos productos efectivos para hacer frente a las EO) tiene como consecuencia la muerte de miles de personas cada año y la degradación de las condiciones de vida de muchas otras debido a la enfermedad, el debate sobre la ética de esta «omisión» debe ser, cuanto menos, planteado, discutido y argumentado de forma diligente por parte de los agentes implicados. Estos agentes son: gobiernos, industria farmacéutica y afines, organizaciones que defienden los derechos de los más débiles y la sociedad en general. No se trata aquí de exigir o pedir a la industria que cambie completamente el modelo de «máximo beneficio» que hasta ahora tan rentable le ha resultado, sino, simplemente, plantear que con un mínimo esfuerzo por parte de la industria farmacéutica, la calidad de vida de millones de personas en el mundo mejoraría de forma significativa.

Razones para una mayor implicación de la industria farmacéutica en las EO: Las razones que sustentan la oportunidad de una mayor implicación ética de la industria farmacéutica son varias, a saber:

- Los países occidentales, en los que las principales industrias farmacéuticas tienen sus sedes, empezaron a crear a partir de la época colonial un sistema de relaciones en el que el gran beneficiado era el país occidental, mientras que el gran perjudicado (extracción de recursos naturales, esclavitud etc.) era el país colonizado. Si bien la situación mejoró sensiblemente con la paulatina abolición de las colonias, lo cierto es que el sistema imperante a día de hoy (a través del Fondo Monetario Internacional, FMI, o el Banco Mundial, BM, y otras instituciones) continúa exprimiendo los países en vías de desarrollo a través de planes de ajuste estructurales impuestos como condición de los préstamos concedidos. La industria farmacéutica ha sido una de las industrias que mayor provecho económico ha sacado de esta situación, tanto en la época de las colonias, como a día de hoy (Global Issues, 2011). 
- Teniendo en cuenta el importante porcentaje de fondos públicos que se destinan a la industria farmacéutica en concepto de I+D (ya sea de forma directa, o bien con ventajas y beneficios fiscales que se le permiten), la industria farmacéutica no tiene la autoridad moral para decidir quién tiene acceso y quién no al uso de ciertos medicamentos en base a unos precios sustentados por la política del «máximo beneficio» (Global Issues, 2011). Es decir, con recursos en parte públicos y en parte privados, la industria farmacéutica construye un modelo de negocio basado en el máximo beneficio privado. Parecería lógico esperar que, debido a la importante participación de fondos públicos (de la forma que sean), las instituciones y gobiernos implicados tuvieran la oportunidad de opinar e influir sobre las políticas de investigación que las empresas eligen, pudiendo desembocar esto en una mayor inversión en la investigación de las EO no sujeta al retorno económico estricto característico de estas grandes corporaciones.

- Aun decidiendo aportar cierta cantidad de los beneficios a investigar las EO, las políticas legales que las grandes corporaciones defienden a nivel global (patentes, propiedad intelectual etc.) impide que muchos de estos países puedan tener acceso a ciertos medicamentos genéricos a un precio acorde al nivel económico del país destinatario (Global Issues, 2011).

- Lefebvre (Lefebvre, 2006) defiende la responsabilidad moral que la industria farmacéutica tiene en un tema tan importante como la medicina. El autor entiende que todo negocio necesita un retorno económico, pero se cuestiona cuál es el precio que se paga para ello: «Sin lugar a dudas, negocios y corporaciones deben generar beneficios con el fin de mantenerse en el mercado. De todos modos, es realmente injusto terminar con los medios de vida de una persona en el intento de mejorar los resultados unas décimas de porcentaje o anticiparse a los resultados de un mal cuatrimestre».

A diferencia de otros sectores en los que mayor innovación significa sobre todo mejorar en comodidad y estilo de vida, por ejemplo el sector del automóvil, en el caso del sector farmacéutico mejorar en inversión en I+D significa salvar vidas y mejorar las condiciones de vida de la población. Encajando el problema de las EO en este escenario, el problema se vuelve más urgente desde un punto de vista ético, puesto que mejorando la inversión en I+D, se puede salvar y mejorar la calidad de vida de millones de personas. Por esta razón, la responsabilidad ética de la indústria farmacéutica y de los lobbys que las defienden, especialmente en los EEUU, debería ser suficiente para plantear algunos cambios en el sistema actual que rige el negocio farmacéutico a nivel mundial. Está muy bien y es muy lógico que directivos, asesores etc. busquen maximizar el beneficio de la empresa, pero cuando para llevar a cabo estas políticas se está limitando el potencial investigador y de desarrollo de forma evidente y consciente, se está entrando en un dilema ético que merece una reflexión más profunda.

Algunos autores, por ejemplo Pogge (Pogge, 2005), afrontan este dilema ético de una forma simple y directa: gobiernos y ciudadanos de los países desarrollados tienen (tenemos) el deber moral de mejorar el acceso de los países menos desarrollados a los avances médicos, debido a que somos en parte responsables de la actual situación que sufren estos países al colaborar, de forma más o menos intencionada, en el establecimiento y mantenimiento del 
orden global que posibilita esta situación. Otros autores del ámbito médico opinan en el mismo sentido, aunque apunten a nivel de los gobiernos que prestan el dinero a la industria como máximos responsables (Macip, 2010): «(...) las prioridades de los gobiernos no siempre coinciden con los intereses globales. Además, los países donde el impacto de las infecciones es más grave son también los que menos recursos pueden dedicar a la investigación. Por esto hace falta que los países ricos hagan el esfuerzo de subvencionar programas que se puedan aplicar a África, Asia o América del Sur».

\section{Algunas propuestas para mejorar la inversión en investigación biomédica presenta- das hasta la fecha: propuestas de Pogge, Reiss y De Winter}

Tal y como ha quedado patente en los apartados precedentes, la inversión en investigación enfocada a las EO resulta, cuanto menos, insuficiente. Numerosos autores han presentado ideas y propuestas para reformar el sistema actual, deficitario en investigación biomédica para las EO, con el objetivo incentivar la producción científica y técnica que ayude, en un primer estadio, a controlar las EO y, en un segundo estadio y para algunas EO en concreto, a eliminarlas del mundo. Dos de los autores más prolíficos y que mayor debate han suscitado han sido Thomas Pogge y Julian Reiss. A continuación, se analizan de forma resumida los ejes principales de sus propuestas para mejorar la investigación biomédica para las EO.

La propuesta de reforma de Thomas Pogge (Pogge, 2005) parte de una idea simple y directa: «La participación en la imposición de reglas sociales constituye una violación de los derechos humanos sólo cuando estas reglas, de forma previsible y evitable, privan a los seres humanos de acceso seguro de los derechos humanos. (...) Los gobiernos y ciudadanos de los países desarrollados pueden y deben saber que la mayor parte de la morbilidad y mortalidad prematura se puede evitar con reformas sencillas y factibles, entre otras, la reforma global del sistema de salud». Pogge concluye que el conocimiento médico debe ser libremente accesible como un bien público global. Para ello, propone que cada nuevo fármaco se recompense en proporción a su impacto sobre la enfermedad entendida de forma global y no a exclusivamente través de incentivos basados en los beneficios que genera la explotación monopolística de un medicamento, protegida por el régimen de patentes. Pogge considera su propuesta como un «complemento al sistema de patentes actual» asegurando que, si se implementa de forma adecuada, esta propuesta reducirá los efectos negativos creados por el sistema de patentes (Pogge, 2008). En todo caso, y si bien Pogge no propone le eliminación total del sistema de patentes, sí tiene muy claro éste no es un sistema válido para el acceso a los medicamentos de los más pobres (Pogge, 2012a).

Para llevar la idea a la práctica, Pogge propone la creación del Health Impact Fund (HIF, 2012), que se puede resumir en 3 ideas básicas:

1. El resultado de desarrollar nuevos fármacos esenciales debe provenir de fondos públicos. Todas las compañías farmacéuticas tienen derecho al uso de dichos fondos.

2. La firma que desarrolle el fármaco será recompensada según el impacto del descubrimiento en la salud global.

3. La contribución a los fondos por parte de las distintas Naciones será justa, factible y políticamente realista. 
En una reciente revisión de su propuesta (Pogge, 2012b), Pogge resume en tres puntos los beneficios básicos que se obtendrían después de la implementación del HIF: Primero, dirigir la innovación farmacéutica hacia las enfermedades más relevantes, incluyendo aquí las EO; segundo, el coste de los medicamentos obtenidos bajo el paraguas del HIF es sensiblemente inferior al obtenido bajo el sistema de patentes; tercero, se promueve el uso óptimo de los medicamentos registrados bajo el HIF por parte de sus desarrolladores.

Si bien Julian Reiss (Reiss, 2009) comparte con Pogge una parte de sus ideas, cree que sus propuestas pueden cambiar el sistema de forma mínima y, por tanto, tendría un impacto práctico más bien pobre.

Reiss considera que los siguientes puntos considerados en el HIF propuesto por Pogge no están del todo resueltos: 1- Los costes: En la propuesta planteada por Pogge, se sigue considerando como válido un sistema de patentes que tiende a inflar el precio de los medicamentos. La crítica que hace aquí Reiss es que, según la propuesta de Pogge, los costes asociados que deberán pagar los gobiernos a la industria en compensación al impacto sanitario de los productos desarrollados será superior al necesario debido a que los productos desarrollados bajo este sistema (HIF) competirán en un mercado con los productos desarrollados bajo el sistema de patentes y, por tanto, en un mercado de precios inflados; 2- La financiación: no se propone una solución para financiar los 45-90 billones USD anuales que se estima costará la creación del HIF, solamente se argumenta que los gobiernes occidentales «deberían» pagarlo. En este sentido, el propio Pogge asume que será necesario mucho tiempo para conseguir la financiación por parte de los gobiernos para implantar el HIF (Pogge, 2008); 3- Eficiencia del sistema: con la aplicación del HIF, las distintas empresas se lanzan a una «carrera» para desarrollar el producto con mayor impacto en la sanidad global y, por supuesto, ser recompensados al final por ello. La crítica de Reiss en este punto radica en el hecho de que, presumiblemente y con vistas al premio económico que espera al final del camino, las empresas no compartan los resultados preliminares y parciales que se van obteniendo en el proceso de investigación, ralentizando y disminuyendo la eficiencia del mismo; 4- Los tratamientos: la aplicación del HIF limita la investigación de aquellas prácticas no patentables, por ejemplo la higiene en ciertas enfermedades etc.; 5- La medición: Para Reiss, resultaría muy difícil valorar el impacto que un nuevo desarrollo pueda tener sobre la sanidad global de una forma objetiva. Pogge y otros defensores del HIF son plenamente conscientes de la dificultad de implementar el HIF a nivel práctico. Así lo indican en un trabajo de 2011 (Grootendorst, 2011), proponiendo una prueba a pequeña escala del HIF, por ejemplo con un solo producto y en un solo país o región, para seguir con experimentos sociales aleatorizados con el fin de evaluar el éxito o no de esta propuesta. En trabajos posteriores (Pogge, 2012a), Pogge ha planteado incluso la idea de que este sistema podría funcionar en otros ámbitos diferentes al de la innovación farmacéutica, por ejemplo en el campo de la agricultura.

Analizadas las críticas al planteamiento de Pogge, la propuesta de Reiss reposa sobre 2 conceptos bien diferenciados:

1. Reforma de la organización de la BMR con objeto de mejorar varios errores y deficiencias del sistema actual; especialmente una reducción gradual del tiempo útil de las patentes y de aspectos patentables de las investigaciones. Según Reiss, la industria farmacéutica se aprovecha de la propiedad intelectual para desarrollar fármacos con 
mejoras que tan solo son sutiles y para crear nuevos mercados en lugar de innovar realmente, especialmente frente a las EO. Para aprobar un fármaco, las pruebas deberían ser externas a la empresa y reguladas por entes públicos subvencionados a través de un «club» al que las empresas miembros pagan una cuota de socio.

2. Crear un sistema de incentivos para redireccionar las prioridades de la BMR hacia el ideal de principio justo y equitativo. Reiss propone aquí la creación de un instituto global de la salud que subvencione y regule la investigación de las EO.

En estas propuestas de Reiss, se distingue claramente la influencia y el trabajo conjunto realizado con su colega Philip Kitcher, especialmente las ideas que se desprenden de la que Kitcher denominó «Well-Ordered Science», la ciencia bien ordenada (Kitcher, 2003). Según esta idea, la ciencia solamente estará bien ordenada si las investigaciones se dirigen siguiendo el principio de «bien común», entendido este como la investigación orientada en base a deliberaciones democráticas llevadas a cabo por participantes suficientemente informados y aptos en el terreno de discusión dado, que plantean de forma conjunta las necesidades y aspiraciones del resto de ciudadanos. La investigación se orienta con el objetivo de reducir la carga que supone una enfermedad siguiendo el principio de la «parte justa»: a igualdad de probabilidades de éxito para dos investigaciones, se destinarán los recursos a aquella orientada a combatir la enfermedad que mayor sufrimiento humano cause.

Para conseguir estos objetivos, Kitcher propone un itinerario marcado por puntos a tener en cuenta: primero, realizar compromisos para proyectos específicos y con los recursos (dinero) establecidos. Segundo, la búsqueda más eficiente de los proyectos acordados se determina sin perjuicio de las restricciones morales. Tercero, la aplicación práctica de los resultados de los estudios la discutirán los «deliberantes» (expertos de distintos ámbitos y posicionamientos que no tienen que ser necesariamente científicos) que, una vez determinadas las empresas ideales que participarán en el proyecto, pondrán en marcha un plan de acción sin dejar de tener en cuenta las opiniones de grupos minoritarios.

Las ideas de «principio justo y equitativo» es una de las principales ideas de Reiss que se fundamentan en la «Well-Ordered Science».

Por último, la propuesta de Jan de Winter (De Winter, 2011) tiene como objetivo financiar la investigación de forma que se obtenga el mayor impacto sanitario con el mínimo coste de dinero público. Para conseguir esto, de Winter propone incrementar la financiación de los gobiernos a las ONGs con el fin de que estas promuevan la sanidad de los países en vías de desarrollo. La novedad en este apunto radica en que de Winter propone que la financiación de los gobiernos se haga específicamente a aquellas ONG más exitosas. Algunos ejemplos son, según de Winter, Drugs for Neglected Diseases Initiative (DNDi), Medicines for Malaria Venture (MMV) y the TB Alliance, entre otras. Estas organizaciones actuarían como administradores de los fondos recibidos por parte de los gobiernos. La opción que plantea de Winter es que estas ONG abran la puerta a la promoción de otros aspectos de la sanidad que no sean, necesariamente, el desarrollo de nuevos fármacos para las EO. Con el fin de evitar el desvío de fondos que nunca llegarán a buen puerto por parte de los gobiernos, de Winter propone aprobar las ayudas solamente en aquellos casos en que se haya documentado y asegurado que el resultado será innovador, que se finalice el proceso de investigación y, sobretodo, que los resultados sean aplicados de forma práctica en los países en vías de desarrollo. De Winter 
concluye su propuesta defendiendo que este modelo puede suplantar el modelo actual, basado en la investigación (con fondos públicos) en universidades y en el que las empresas farmacéuticas entran en escena contribuyendo con la I+D en aquellos proyectos que puedan tener interés comercial. De Winter asume que es necesaria mayor experiencia por parte del sistema si se quiere aplicar este modelo, puesto que hasta el momento es un modelo solamente teórico.

\section{Una propuesta alternativa}

La industria farmacéutica es uno de los sectores con mayores beneficios de la economía occidental. Reiss (2009), lo describe con un gráfico ejemplo. En EEUU (2002) los beneficios de las 10 principales empresas farmacéuticas (35,9 billones USD) incluidas en la lista «Fortune 500» (ranking de las 500 principales empresas estadounidenses de capital abierto a cualquier inversor) fue mayor que el beneficio obtenido por las restantes 490 empresas que componían la lista (33,7 billones USD). El negocio farmacéutico es pues, un negocio muy lucrativo. Esta desproporción tiene múltiples explicaciones, entre las que podemos citar las siguientes. Buena parte de estos lucrativos beneficios se consiguen gracias a los resultados económicos que se generan a raíz de un proceso de investigación exitoso protegido por el sistema de patentes, sistema al que no pueden atenerse (o que se presentan con unas condiciones mucho menos favorables) otros sectores que también invierten importantes recursos en I+D, por ejemplo, el sector del automóvil, aeroespacial o tecnológico. Además, y como ya hemos visto, las empresas farmacéuticas reciben importantes cantidades de fondos públicos destinadas a I+D con la finalidad de mejorar la salud de los ciudadanos. Pero el hecho es que la mayor parte de estos fondos se destinan a desarrollar productos que no son necesarios o que aportan solamente ligeras variaciones a productos ya existentes en el mercado (Reiss, 2010). $\mathrm{Si}$, tal y como se ha demostrado, existe una falta real de investigación en ciertas enfermedades (por ejemplo, las EO), podría plantearse el hecho de invertir estos fondos públicos, al menos una parte de ellos, en la investigación de aquellas enfermedades que realmente lo necesitan.

Sin el aporte de estos fondos públicos, el problema moral y ético quedaría restringido al ámbito de la RSC que cada empresa en particular quiera aplicar, puesto que las normas que rigen nuestra sociedad occidental, tanto legales como morales, permiten (y de hecho en muchos casos premian) a aquellas empresas que maximizan sus beneficios, a veces sin importar mucho el camino seguido hasta conseguirlo. El dilema ético se acentúa en gran medida cuando se inyectan estos fondos públicos que provienen de distintos Estados, Organismos Internacionales etc. y que tienen como fin incentivar la inversión en BMR de la industria farmacéutica para mejorar la calidad de vida de la población. Aunque puedan existir lecturas divergentes, es evidente que la sanidad en los países desarrollados ha mejorado, en parte gracias a la industria farmacéutica. Pero en el caso de los países en vías de desarrollo, la mejora está a años luz de lo que sucede en Occidente. La cuestión ética que se plantea en este punto es la siguiente. En un mundo cada día más globalizado, ¿tienen la industria farmacéutica, y los financiadores públicos cuyos fondos ella gestiona, una responsabilidad ética sobre la inversión en investigación de las enfermedades olvidadas?

La propuesta presentada en este apartado responde a la siguiente idea compartida con varios autores (Pogge, 2005): los países desarrollados, su industria farmacéutica y sus ciudadanos en general tienen una responsabilidad ética sobre el impacto que la pobreza 
y, en el caso que nos ocupa, las EO tienen sobre los ciudadanos de los países en vías de desarrollo. Ésta responsabilidad viene dictada por dos motivos básicos:

1. Motivos humanitarios: La parte de la población que dispone de mayores recursos tiene el deber moral de reducir en la medida de lo posible el sufrimiento de la parte de la población con menos recursos. Económicamente, es posible mejorar algunos de los aspectos que más lastran y castigan a los más desfavorecidos; por ejemplo, incidir de una forma mucho más diligente sobre el impacto que las EO tienen mejorando el conocimiento científico sobre las mismas.

2. Motivos históricos: Si no todos, la inmensa mayoría de países desarrollados se ha beneficiado, en los momentos históricos de mayor crecimiento económico, de los países en vías de desarrollo y/o de sus habitantes; por ejemplo colonias, explotación de recursos naturales, esclavitud etc. Los países occidentales y su industria farmacéutica, como estandarte del desarrollado grado de salud conseguido por sus ciudadanos, tienen una responsabilidad social con los países que colaboraron (de forma voluntaria o no) al desarrollo de sus economías. Esta es una oportunidad de oro para devolver, al menos en parte, los beneficios y desarrollo que los países pobres aportaron a los países ricos.

Definición del escenario: El futuro a medio plazo no parece muy alentador para mejorar el grave impacto que las EO tienen sobre la mortalidad y la calidad de vida de una parte muy importante de la población mundial: las previsiones de incremento mundial de la población, hasta 9.300 millones en 2050 (UN, 2011), unido a la escasez de recursos energéticos y alimentarios cada vez mayor, presentan un horizonte ciertamente gris en el objetivo de reducir el impacto que las EO tienen en los países en vías de desarrollo.

Para mejorar la inversión de recursos en investigación de las EO, es de vital importancia definir primero cuál o cuáles son las enfermedades que deben ser objeto de tales inversiones. Dentro del heterogéneo grupo de EO, hay algunas de ellas para las que la inversión en BMR no es tan determinante para su control y/o erradicación como para otras enfermedades. Por ejemplo, en el caso de las helmintiasis (enfermedades de origen parasitario que pueden afectar distintas partes del cuerpo), existen opciones diferentes a la inversión en BMR que pueden dar resultados prácticos más que satisfactorios con un coste inferior; por ejemplo, incrementando las medidas de higiene. Además, y para estas enfermedades en concreto, existen ya fármacos eficaces (por ejemplo avermectinas, bencimidazoles etc.). El reto en este punto, más que desarrollar nuevos fármacos, sería incentivar un plan de higiene para las poblaciones locales y facilitar el acceso a los fármacos ya existentes y, en muchos casos, con un coste ciertamente asequible, ya que muchos de ellos se usan incluso en medicina veterinaria.

Así pues, el primer paso sería definir qué enfermedades precisan de inversión en BMR de forma más urgente y establecer una lista de enfermedades prioritarias, basando dicha lista en:

- Índice DALY.

- Distribución de la enfermedad (enfermedad ampliamente distribuida o localizada en una población). 
- Previsiones a medio plazo de la evolución de la enfermedad, según índices como la incidencia, la prevalencia, la mortalidad etc.: Evidentemente, algunas enfermedades merecen actuaciones mucho más urgentes que otras.

El objetivo de esta clasificación es centrar los recursos disponibles (económicos, de personal y de tiempo) en aquellas enfermedades en las que el retorno de la inversión sea más rápido y, sobre todo, más efectivo en lo que a la calidad de vida de las personas afectadas (o en riesgo) se refiere. Una vez definido este punto, será necesario mejorar la inversión que las diversas instituciones y, sobretodo, la industria privada destinan a las EO.

Propuesta de mejora de la inversión en BMR de las EO: Como objetivo básico de la propuesta, se pretende mejorar la inversión en BMR de las EO pre-definidas como prioritarias, haciendo hincapié sobre dos pilares básicos:

1. Reducir parte de los privilegios económicos de la industria farmacéutica de los países avanzados.

2. Impulsar la colaboración con la industria farmacéutica de países emergentes, como pueden ser China, India o Brasil.

Para conseguir este objetivo, no se trata de penalizar directamente a la industria de los países desarrollados, que tiene su derecho a invertir sobre las enfermedades y los productos comerciales que mayores beneficios le aportan, sino de reducir los privilegios económicos que reciben de los fondos públicos. Por ejemplo, no se trata en este caso de modificar la ley de patentes etc., sino simplemente de reducir las ventajas que la industria recibe a modo de ahorro a nivel de impuestos; no se trata de modificar ni las leyes que rigen el registro y comercio de medicamentos, sino simplemente «recuperar» parte del dinero que la industria farmacéutica ahorra en forma de impuestos que no paga (exención de cierto tipo de impuestos, ayudas estatales), bonos en concepto de investigación, ayudas etc. y que reinvierte en el desarrollo de productos que ya le aportarán cuantiosos beneficios al ser concebidos para su uso en enfermedades de los «países ricos» y destinados a segmentos de la población con un poder adquisitivo medio-alto. El objetivo final es pues invertir este dinero «recuperado» en la investigación de las EO; invertir el dinero en aquellas enfermedades en las que pocas (o ninguna) empresas privadas invertirán ya que no le sacarán un rédito económico suficiente (a juicio suyo).

Así pues, la propuesta para redefinir la inversión en investigación de las EO, se compone de los puntos que siguen:

1. La bolsa de dinero: Crear una bolsa de dinero para financiar los costes derivados de la inversión en investigación destinada a las EO. El dinero de esta bolsa provendrá, principalmente, de 3 fuentes:

- Industria farmacéutica de los países desarrollados, a través de la recuperación de ciertos impuestos y beneficios de los que hasta ahora estaban exentos, al menos en parte.

- Gobiernos de los países desarrollados.

- Organismos e instituciones mundiales, tanto públicos como privados. 
2. El Organismo Internacional: Creación de un Organismo Internacional que gestione los recursos generados y dirija el destino y los colaboradores para realizar las investigaciones.

3. Contrato de colaboradores para la investigación: La selección de los colaboradores por parte de este Organismo Internacional no debe excluir a ningún agente; por ejemplo, se debe contemplar la posibilidad de que las investigaciones las lleven a cabo tanto la industria privada, bien sean grandes corporaciones o bien pequeñas empresas de biotecnología, como también centros de investigación públicos (universidades etc.) o, por qué no, grupos de colaboración entre varios de estos agentes. Así pues, el Organismo Internacional sería el promotor de un «contrato de obra y servicio» para estos agentes con el fin de que desarrollen un proyecto de investigación definido y concreto. En este ámbito, algunos trabajos ya han apuntado opciones para el desarrollo de fármacos para las EO que incluyen sociedades entre empresas innovadoras de países en vías de desarrollo con proyectos público-privados de los países desarrollados (Chaudhuri, 2010) o bien la opción de que la investigación se lleve a cabo íntegramente por empresas de países emergentes, por ejemplo India (Kettler, 2001).

4. Producción comercial en países emergentes: El Organismo Internacional es quién dispone de toda la información y los derechos de uso de los resultados de las investigaciones llevadas a cabo. Es éste organismo quien decide la mejor forma de aplicar los conocimientos teóricos adquiridos a la práctica; es decir, plasmar la teoría en frutos tangibles de los que se puedan servir los países en vías de desarrollo. Los países emergentes, por ejemplo India, China y Brasil y, especialmente su floreciente industria farmacéutica, suponen el eslabón intermedio entre los países desarrollados y los países en vías de desarrollo; combinan, en cierto modo, el «conocimiento» de los países ricos con las «necesidades» de los países pobres. Algunos estudios (Murdock, 2011) remarcan la capacidad que tiene la industria local de los países emergentes para satisfacer este nicho de mercado. Se trata pues, de implicar a la industria de estos países para producir a escala comercial los productos desarrollados. Al no existir patentes ni intereses comerciales más allá de los que rigen los contratos de investigación (primero) y de producción (después), el coste de producción se reduce de forma sensible si lo comparamos con un fármaco que se rige por las leyes de patentes «tradicionales» e intereses comerciales puramente privados. Es evidente que la industria de los países emergentes tiene unos costes de producción menores, por ejemplo, el coste de producción medio de un fármaco en la India es un 50\% inferior al coste de producción en Europa o EEUU; las empresas locales conocen esta ventaja y ven como una oportunidad de negocio el contrato de parte de la producción de las empresas internacionales (Deutsche Bank Research, 2008). Se trata de aprovechar este punto junto con el interés de estos países/industria en producir y comercializar un fármaco con unos márgenes económicos adecuados y con un potencial de consumo que se verá elevado a medida que baja el coste del medicamento: más gente estará dispuesta (y podrá) pagar por un fármaco a medida que el coste del mismo sea menor, tornándose esta opción más semejante a una economía de escala (mayor volumen con menor margen). Las conclusiones de un informe del Office of Health Economics (Towse, 2011) ya apuntan en este sentido: «Con un diferencial de precio, 
los mercados de los países en vías de desarrollo no son necesariamente poco provechosos, sino que serán una fuente de crecimiento para las empresas multinacionales y las empresas regionales». En este punto de la propuesta es imprescindible el riguroso control por parte del Organismo Internacional de las condiciones laborales y humanas de producción por parte de los trabajadores de las empresas de los países emergentes. Es imperativo que el contrato establezca unas condiciones de trabajo mínimas y dignas para los asalariados con el fin de evitar la explotación laboral que, con cierta frecuencia, se observa en estos países.

\section{Conclusión}

El impacto que las enfermedades olvidadas tienen sobre una parte muy importante de la población mundial es innegable y evidente. En la mayoría de EO, un moderado esfuerzo en inversión en I+D supondría una mejora significativa para la calidad de vida de muchas personas. Hasta el momento, la colaboración en I+D para las EO de la industria farmacéutica de los países desarrollados ha sido muy limitada y ha quedado muy por debajo de su potencial real. Mientras, esta misma industria se está beneficiando de ayudas con fondos públicos y está centrando su principal negocio en el desarrollo de productos para enfermedades de los países desarrollados. La responsabilidad ética que estas empresas, los gobiernos y los ciudadanos de los países desarrollados tienen sobre el control (y erradicación) de las EO en los países en vías de desarrollo no deja margen a perder más tiempo y desperdiciar más recursos. Una mayor implicación, especialmente de los gobiernos y de la industria farmacéutica de los países desarrollados, exige un cambio en la forma de gestionar la inversión en I+D para las EO. Numerosos autores han presentado hasta la fecha sus propuestas, algunas de ellas con más acierto que otras. En este trabajo, se presenta otra propuesta que tiene como ejes centrales reducir los privilegios de la industria farmacéutica de los países desarrollados e incrementar la colaboración con la naciente y potencial industria farmacéutica de los países emergentes, industria que al fin y al cabo, es más cercana a la población en riesgo de sufrir las consecuencias de las EO.

\section{Bibliografía}

Boldrin, Michele y Levine, David K (2008): Against intellectual monopoly (chapter 9). Cambridge University Press.

Chaudhuri, Sudip (2010): R\&D for development of new drugs for neglected diseases in India. Int. J. Technology and Globalisation, Vol. 5, Nos. 1/2, 2010.

Deutsche Bank Research (2008): Current Issues Asia. India's pharmaceutical industry on course for globalization. Deutsche Bank AG, DB Research, Frankfurt am Main. Internet and e-mail ISSN 1612-3158.

De Winter, Jan (2011): How to Make the Research Agenda in the Health Sciences Less Distorted. Theoria 72: 75-93.

FARMAINDUSTRIA (2004): La industria farmacéutica en cifras. http://www.farmaindustria.es/Farma_Public/Publicaciones/index.htm (consultado 05/05/2011).

Global Issues. http://www.globalissues.org/article/52/pharmaceutical-corporations-andmedical-research (consultado 17/09/2011). 
Grootendorst, Paul et al. (2011): New approaches to rewarding pharmaceutical innovation. CMAJ, April 5, 183(6):681-685.

Health Impact Fund. http://www.healthimpactfund.org/wp-content/uploads/2012/11/HIFshort_sp.pdf

International Federation of Pharmaceutical Manufacturers \& Associations (2007): News release. Updated IFPMA survey shows growing pharmaceutical industry contribution to improving developing world health. Geneva, 2 November 2007.

Ketter, Hanna E.and Modi, Rajiv (2001): Building local research and development capacity for the prevention and cure of neglected diseases: the case of India. Bulletin of the World Health Organization, 79 (8), 2001.

Kitcher, Philip (2003): Science, truth and democracy. Oxford University Press 2003. ISBN13: 9780195165524.

Lawford-Smith, Holly (2012): The motivation question: Arguments from justice and from humanity. British Journal of Political Science. Volume 42, Issue 03 (p. 661-678).

Lefebvre, Paul and Miller, Gerald (2006): Pharmaceutical giants and corporate social responsibility: A hard pill to swallow. www.rockhurst.edu/news/events/images/projecti/ lefebvre.pdf (consultado 17/09/2011).

Leisinger, Klaus M. (2005): The corporate social responsibility of the pharmaceutical industry: Idealism without illusion and realism without resignation. Business ethics Quaterly. Volume 15, Issue 4 (p. 577-594). ISSN 1052-150X.

Light, Donald W. (2006): Basic research funds to discover important new drugs: who contributes how much? In: M.A. Burke (de.). Monitoring the financial flows for health research 2005: Behind the global numbers. Geneva, Switzerland: Global Forum for Health Research, pp. 27-43.

Light, Donald W. and Warburton, Rebecca (2011): Demithologizing the high costs of pharmaceutical research. Biosocieties 6 (1): 34-50.

Macip, Salvador (2010): Les gran epidèmies modernes: La lluita de l'home contra els enemics invisibles. Edicions La Campana, Barcelona, 2010. ISBN: 978-84-96735-40-8.

Mathers, Colin D. et al. (2007): Measuring the Burden of Neglected Tropical Diseases: The Global Burden of Disease Framework . PLOS Neglected tropical diseases 1 (2):e114.

Murdock, Mitchell (2011). The growth of international pharmaceuticals. Yale journal of medicine and law: An undergraduated publication, Vol. VII, Issue 2, 2011.

Oxfam, Save the Children, VSO (2002): Beyond philantropy: the pharmaceutical industry, corporate social responsibility and the developing world. An Oxfam, save the Children and VSO joint report. Editors: Bonita Glanville Morris, Oxfam GB Creative Services Unit, 2002.

Pogge, Thomas W. (2005): Human rights and global health: A research program. Metaphilosophy 36 (1/2): 182-209.

Pogge, Thomas W. (2008): Access to Medecines. Public Health Ethics, Vol. 1, Number 2, 2008: 73-82.

Pogge, Thomas W. (2012a): Results based financing: The «Health Impact Fund» as an example for smart pro-poor innovations. KFW-Development Research Meinungsforum Entwicklungspolitik, Nº1, 16 January 2012. 
Pogge, Thomas W. (2012b): The Health Impact Fund: Enhancing justice and efficiency in global health. Journal of human development and capabilities: A multidisciplinary Journal for people-centered development, 13:4, 537-559.

Pollock, Anne (2011): Transforming the critique of big pharma. Biosocieties 6 (1): 106-118.

PubMed, U.S. National Library of Medicine National Institutes of Health. http://www.ncbi. nlm.nih.gov/pubmed (14/07/2011).

Reiss, Julian and Kitcher, Philip. (2009): Biomedical research, neglected diseases, and wellordered science. Theoria 66: 263-282.

Reiss, Julian (2010): In favour of a millian proposal to reform biomedical research. Synthese 177: 427-447.

The New York Times (11/10/2011): http:/query.nytimes.com/search/sitesearch?date_select $=$ full $\&$ query $=\&$ type $=$ nyt $\& x=13 \& y=10$

Towse, Adrian et. al. (2011): Drugs and vaccines for developing countries. Office of Health Economics. Ocasional paper 11/04. Office of Health Economics, London, 2011.

Troullier, Patrice et al. (2002): Drug development for neglected diseases: a deficient market and a public-health policy failure. The Lancet 359: 2188-2194.

United Nations (1999): United Nations conference on trade and development. The social responsibility of transnational corporations. United Nations, New York and Geneva, 1999.

United Nations Press release, 3 May 2011: http://esa.un.org/unpd/wpp/index.htm (03/06/2011).

Vanderelst, Dieter and Speybroeck, Niko (2010): Quantifying the lack of scientific interest in negelcted tropical diseases. PLOS Neglected tropical diseases 4 (1): e576.

World Health Organization (2009): Neglected tropical diseases, ancient companions of poverty. Flyer WHO/HTM/NTD/2009.1 . WHO 2009.

World Health Organization (2010): First WHO report on NTD: Working to overcome the global impact of neglected tropical diseases. WHO 2010. ISBN 9789241564090. 